\title{
Stochastic inflation with quantum and thermal noise
}

\author{
Z. Haba ${ }^{\mathrm{a}}$ \\ Institute of Theoretical Physics, University of Wroclaw, 50-204 Wrocław, Poland
}

Received: 28 November 2017 / Accepted: 16 July 2018 / Published online: 24 July 2018

(C) The Author(s) 2018

\begin{abstract}
We add a thermal noise to Starobinsky equation of slow roll inflation. We calculate the number of e-folds of the stochastic system. The power spectrum and the spectral index are evaluated from the fluctuations of the e-folds using an expansion in the quantum and thermal noise terms.
\end{abstract}

\section{Introduction}

The standard $\Lambda \mathrm{CDM}$ model describes the evolution of the universe in agreement with observations [1]. The fast expansion at the early stages of the evolution can be explained in terms of a scalar field (inflaton). A quantization of the scalar field and gravitational perturbations leads to fluctuations which can explain structure formation and the power spectrum of density fluctuations in the universe [2-7]. The model is introducing some new (dark) forms of matter and energy which are not interacting with the inflaton. If we assume that there are some interactions of the inflaton with the unknown forms of matter then the wave equation for the inflaton is transformed into a stochastic equation which in a flat expanding metric (with the scale factor $a$ and $H=a^{-1} \partial_{t} a$ ) takes the form

$$
\begin{gathered}
\partial_{t}^{2} \phi-a^{-2} \triangle \phi+\left(3 H+\gamma^{2}\right) \partial_{t} \phi \\
+V^{\prime}(\phi)+\frac{3}{2} \gamma^{2} H \phi=\gamma a^{-\frac{3}{2}} \eta
\end{gathered}
$$

where $\gamma^{2}$ is a friction related to the Gaussian noise

$$
\langle\eta(t) \eta(s)\rangle=\delta(t-s)
$$

according to the fluctuation-dissipation relation. Equation (1) has been derived in [8] (see also [9]). The friction $\gamma^{2}$ is proportional to temperature. The noise $\eta$ comes from the thermal (Gibbs) distribution of the initial positions and velocities of the particles of the environment (in general, the environment may consist of any degrees of freedom which are unobservable and averaged in a description of an interaction with $\phi$ ). Equation (1) is a basis of the warm inflation [10]. In such a model the resonant reheating is unnecessary as the temperature during inflation does not fall to zero owing to the creation of radiation as a result of the decay of the inflaton. The quantum fluctuations of the inflaton and gravitational perturbations are usually described $[3,7]$ in a linear approximation. Starobinsky $[4,11]$ (see also [12]) discovered that quantum fields at large time in an expanding universe behave like a classical diffusion process. Then, the high momentum part of the quantum field can be treated as an additional (quantum) noise in the inflaton wave equation. Such a treatment of quantum fluctuations during inflation goes beyond a linear approximation. The quantum noise has been widely studied in Refs. [13-23]. The Fokker-Planck equation for the probability distribution of the inflaton has been explored in detail. In principle, the Fokker-Planck equation contains all the information about the probability distribution. In particular, the power spectrum of fluctuations could be calculated as discussed in $[21,22]$ (see also Appendix B here). However, in [23] (following [11], see also [24,25]) an alternative method has been proposed for a calculation of the power spectrum of the quantum noise based on fluctuations of the e-folds. In this paper we extend the method to the calculation of the power spectrum of the system which contains both the quantum noise and the thermal noise. The plan of this paper is the following. In Sect. 2 we discuss the stochastic equation with the quantum and thermal noises in the slow roll approximation. In Sect. 3 following Refs. [23,26] we obtain general formulas for the expectation values of e-folds and the fluctuations of e-folds (spectral function). Then, in Sect. 4 we discuss approximations leading to some explicit formulas for the spectral function and the spectral index. In Appendices $\mathrm{A}$ and $\mathrm{B}$ we discuss fluctuations in soluble models and the relation between Ito and Stratonovitch stochastic equations.

\footnotetext{
a e-mail: zhab@ift.uni.wroc.pl
} 


\section{Slow roll stochastic equations}

We consider two sources of noise in Eq. (1) the thermal noise $\eta$ and the quantum noise $\eta_{S}[4,11,12]$. The quantum noise comes from the large momentum part (above the Hubble horizon) of the scalar field. It can be considered as a part of the quantum inflaton equation. The thermal noise results from an approximation of the interaction with an environment by a Markov process. In Einstein equations the environment could be represented as dark matter or dark energy if we properly choose the environmental interactions [27-29]. We consider Eq. (1) in the slow-roll approximation

$$
\begin{aligned}
\left(3 H+\gamma^{2}\right) \circ d \phi= & -V^{\prime} d t-\frac{3}{2} \gamma^{2} H \phi d t+\gamma a^{-\frac{3}{2}} \circ d B \\
& +\frac{3}{2 \pi} H^{\frac{5}{2}} \circ d W
\end{aligned}
$$

In Eq. (3) we write $\eta=\partial_{t} B, \eta_{S}=\partial_{t} W$ and assume that $W$ and $B$ are independent Gaussian variables. We use the notation $\circ d W$ (after [30]) for the Stratonvitch interpretation of the stochastic differential and the conventional notation of the differential in the Ito interpretation. The difference between Ito and Stratonovitch integrals consists in a different discrete time approximations of the Riemann sums approximating the integral. The Stratonovitch integral $\int f \circ d W$ treats time approximation of $f$ and $W$ (in the Riemann sum) in a symmetric way (so called middle point approximation) whereas in the Ito integral the time in $d W$ is later than in $f$. For an integral with a differentiable function $W$ the various discrete approximations would lead to the same result. However, $W$ is not differentiable. We discuss both interpretations of the stochastic differential for an easy comparison with literature on the subject. The Ito stochastic differential equation can be expressed by the Stratonovitch equation using the rule [30] $f \circ d W=f d W+\frac{1}{2} d f d W$. So, both equations differ by a correction term. The Stratonovitch form is convenient for calculations because it preserves the standard rules of differentiation (the Leibniz rule) [30]. It must be checked in mathematical models which form of the stochastic equation better describes physical processes.

In order to simplify further discussion we assume that $3 H>>\gamma^{2}$ and $V^{\prime}>>\frac{3}{2} \gamma^{2} H \phi$. The friction term $\gamma^{2} \partial_{t} \phi$ is usually related to the decay of the inflaton into radiation [31]. The omission of the $\gamma^{2}$ terms on the lhs of Eq. (1) means a negligible density of radiation (which can be true during inflation [32]). Now, the stochastic equation (3) reads

$$
\begin{aligned}
d \phi= & -\frac{1}{3 H} V^{\prime} d t+\frac{\gamma}{3} a^{-\frac{3}{2}} H^{-1} \circ d B(t) \\
& +\frac{1}{2 \pi} H^{\frac{3}{2}} \circ d W(t) .
\end{aligned}
$$

The Starobinsky $[11,12]$ slow-roll (quantum) system corresponds to the limit $\gamma \rightarrow 0$ of Eq. (4). In order to obtain an agreement with the inhomogeneous inflaton and gravity perturbations (most easily treated in the uniform curvature gauge $[33,34]$ ) we must change the world time $t$ into the efolding time $v$ [21-23] (usually denoted by $N$; we change notation for typographical reasons) describing the change of the scale factor

$v=\int_{0}^{t} H d s=\ln \left(\frac{a}{a_{0}}\right)$.

Now, the diffusion (small roll) system reads

$$
\begin{aligned}
d \phi= & -\frac{1}{3} V^{\prime} H^{-2} d v+\frac{\gamma}{3} a^{-\frac{3}{2}} H^{-\frac{3}{2}} \circ d B(v) \\
& +\frac{1}{2 \pi} H \circ d W(v),
\end{aligned}
$$

together with the differential form of the Friedman equation (taking a derivative in the Friedman equation can allow to treat the environment as a dark energy [27])

$d \ln (H)=-4 \pi G\left(\partial_{\nu} \phi\right)^{2} d \nu$.

We can insert in Eq. (4) either $a(\phi)$ as a function of $\phi$ or $a=a_{0} \exp (v)$ (in such a case we obtain a non-stationary stochastic equation).

In the slow-roll approximation we can derive from Eq. (7) in the no-noise limit

$H=\sqrt{\frac{8 \pi G}{3} V}$,

( $V$ entering Eq. (6) can be determined by $H$ from the wave equation up to an arbitrary constant). Then, from Eqs. (4) and (5) in the no noise limit

$\ln (a)=-8 \pi G \int d \phi\left(V^{\prime}\right)^{-1} V$.

We could take in Eq. (9) the noise into account by means of perturbation methods (the relation between $a(v)$ and $\phi(v)$ will still be discussed in Appendices A and B).

The probability distribution of the solution of Eq. (4) (Stratonovitch interpretation) satisfies the Fokker-Planck equation $[26,30,35]$

$$
\begin{aligned}
\partial_{t} P= & \partial_{\phi} \frac{\gamma^{2}}{18 H a^{\frac{3}{2}}} \partial_{\phi} \frac{1}{H a^{\frac{3}{2}}} P+\frac{1}{8 \pi^{2}} \partial_{\phi} H^{\frac{3}{2}} \partial_{\phi} H^{\frac{3}{2}} P \\
& +\partial_{\phi}(3 H)^{-1} V^{\prime} P .
\end{aligned}
$$

In the Ito interpretation of Eq. (4)

$$
\begin{aligned}
\partial_{t} P= & \partial_{\phi} \partial_{\phi} \frac{\gamma^{2}}{18 H^{2} a^{3}} P+\frac{1}{8 \pi^{2}} \partial_{\phi} \partial_{\phi} H^{3} P \\
& +\partial_{\phi}(3 H)^{-1} V^{\prime} P .
\end{aligned}
$$

If in the e-folding time we treat $a$ as depending on $\phi$ (not on $v)$, then we obtain a stationary form of the Fokker-Planck equation which for the Ito version is 
$\partial_{\nu} P=\frac{\gamma^{2}}{18} \partial_{\phi} \partial_{\phi} \frac{1}{a^{3} H^{3}} P+\frac{1}{8 \pi^{2}} \partial_{\phi} \partial_{\phi} H^{2} P+\partial_{\phi}\left(3 H^{2}\right)^{-1} V^{\prime} P$

and Stratonovitch version

$$
\begin{aligned}
\partial_{\nu} P= & \frac{\gamma^{2}}{18} \partial_{\phi} \frac{1}{(H a)^{\frac{3}{2}}} \partial_{\phi} \frac{1}{(H a)^{\frac{3}{2}}} P+\frac{1}{8 \pi^{2}} \partial_{\phi} H \partial_{\phi} H P \\
& +\partial_{\phi}\left(3 H^{2}\right)^{-1} V^{\prime} P .
\end{aligned}
$$

If we express $a$ by the $v$ time then Eq. (13) reads

$$
\begin{aligned}
\partial_{\nu} P= & \frac{\gamma^{2}}{18} \exp (-3 v) \partial_{\phi} \frac{1}{H^{\frac{3}{2}}} \partial_{\phi} \frac{1}{H^{\frac{3}{2}}} P+\frac{1}{8 \pi^{2}} \partial_{\phi} H \partial_{\phi} H P \\
& +\partial_{\phi}\left(3 H^{2}\right)^{-1} V^{\prime} P
\end{aligned}
$$

It can be seen from Eqs. (6) and (14) that quantum and thermal fluctuations are of the same order if $\gamma^{2} \simeq e^{3 v} H(v)^{5}$. The estimate of the dissipation strength $\gamma$ is relevant for an estimate of the power spectrum and the spectral index at the end of Sect. 4.

Equation (14) does not depend on the $a(\phi)$ approximation. $H(\phi)$ as a function of $\phi$ in Eqs. (10)-(14) can be obtained from Eq. (8). The dependence of $a$ on $\phi$ in Eqs. (10)-(13) in the slow-roll approximation is determined by Eq. (9). Let us consider simple examples. If $V=g \phi^{n}$ (chaotic inflation [36]) then

$\frac{a(\phi)}{a_{0}}=\exp \left(-4 \pi G n^{-1} \phi^{2}\right)$.

If $V=g \exp (\lambda \phi)$ then

$\frac{a(\phi)}{a_{0}}=\exp \left(-\frac{8 \pi G}{\lambda} \phi\right)$,

Note that if $\phi \rightarrow+\infty$ then $a \rightarrow 0$, if $\phi \rightarrow-\infty$ then $a \rightarrow \infty$.

For a flat potential

$V=\frac{\phi^{2}}{K+\phi^{2}}$

we have

$\frac{a(\phi)}{a_{0}}=\exp \left(-2 \pi G \phi^{2}-\frac{\pi G}{K} \phi^{4}\right)$.

For "natural inflation" [37]

$V=g(1-\cos \phi)$.

Then,

$a(\phi)=a_{0} \exp \left(8 \pi G \ln \left(2 \cos ^{2}\left(\frac{\phi}{2}\right)\right)\right.$.

In the case of the double-well potential

$$
\begin{aligned}
V(\phi) & =\frac{g}{4}\left(\phi^{2}-\frac{\mu^{2}}{g}\right)^{2} \\
a & =a_{0}|\phi|^{\frac{2 \pi G \mu^{2}}{g}} \exp \left(-\pi G \phi^{2}\right) .
\end{aligned}
$$

The noise corrected $a(\phi)$ relation could in principle be derived from the solution of Eq. (6) but in general this is possible only on a perturbative level (see Appendix A).

\section{Expectation value of e-folds}

We treat $v$ (in Eq. (5)) as a random time (because $a$ is random). Let us consider a differential of a function of the stochastic process (6) in the e-folding time in the Stratonovitch sense

$$
\begin{aligned}
d f= & \partial_{\phi} f \circ d \phi \\
= & \partial_{\phi} f \circ\left(-\frac{1}{3 H^{2}} V^{\prime} d v+\frac{\gamma}{3(a H)^{\frac{3}{2}}} d B(v)+\frac{1}{2 \pi} H d W(v)\right) \\
= & \partial_{\phi} f\left(-\frac{1}{3 H^{2}} V^{\prime} d v+\frac{\gamma}{3(a H)^{\frac{3}{2}}} d B(v)+\frac{1}{2 \pi} H d W(v)\right) \\
& +\left(\frac{1}{2} \frac{\gamma}{3(a H)^{\frac{3}{2}}} \partial_{\phi} \frac{\gamma}{3(a H)^{\frac{3}{2}}} \partial_{\phi} f+\frac{1}{8 \pi^{2}} \partial_{\phi} H \partial_{\phi} H f\right) d v .
\end{aligned}
$$

For the Ito stochastic equation

$$
\begin{aligned}
d f= & \partial_{\phi} f \circ d \phi \\
= & \partial_{\phi} f \circ\left(-\frac{1}{3 H^{2}} V^{\prime} d v+\frac{\gamma}{3(a H)^{\frac{3}{2}}} d B(v)+\frac{1}{2 \pi} H d W(v)\right) \\
= & \partial_{\phi} f\left(-\frac{1}{3 H^{2}} V^{\prime} d \nu+\frac{\gamma}{3(a H)^{\frac{3}{2}}} d B(v)+\frac{1}{2 \pi} H d W(v)\right) \\
& +\left(\frac{1}{2}\left(\frac{\gamma}{3(a H)^{\frac{3}{2}}}\right)^{2} \partial_{\phi} \partial_{\phi} f+\frac{1}{8 \pi^{2}} H^{2} \partial_{\phi} \partial_{\phi} f\right) d \nu
\end{aligned}
$$

In the rest of this section we follow Refs. [23,26,38]. In the Stratonovitch case we choose a function $f$

$$
\begin{aligned}
& -\partial_{\phi} f_{S} \frac{1}{3 H^{2}} V^{\prime}+\frac{1}{18} \frac{\gamma}{(a H)^{\frac{3}{2}}} \partial_{\phi} \frac{\gamma}{(a H)^{\frac{3}{2}}} \partial_{\phi} f_{S} \\
& +\frac{1}{8 \pi^{2}} \partial_{\phi} H \partial_{\phi} H f_{S}=-1
\end{aligned}
$$

and in the Ito case

$$
\begin{aligned}
& -\partial_{\phi} f_{I} \frac{1}{3 H^{2}} V^{\prime}+\frac{1}{18}\left(\frac{\gamma}{(a H)^{\frac{3}{2}}}\right)^{2} \partial_{\phi} \partial_{\phi} f_{I} \\
& +\frac{1}{8 \pi^{2}} H^{2} \partial_{\phi} \partial_{\phi} f_{I}=-1
\end{aligned}
$$

Then, integrating $d f$ between $v=0$ and $v$ corresponding to the values $\phi(0)=\phi_{i n}$ and $\phi(\nu)=\phi$ we obtain (the expectation value of the Ito integral is equal to zero)

$\langle v\rangle=\left\langle f\left(\phi_{i n}\right)\right\rangle-\langle f(\phi)\rangle$.

Let $\partial_{\phi} f=u$. Equations (24) and (25) for $u$ are of the form

$\partial_{\phi} u+Q(\phi) u=-r$ 
where

$Q=-\frac{1}{3 H^{2}} V^{\prime}\left(\frac{H^{2}}{8 \pi^{2}}+\frac{\gamma^{2}}{18 a^{3} H^{3}}\right)^{-1}$

and (Ito interpretation)

$r=\left(\frac{H^{2}}{8 \pi^{2}}+\frac{\gamma^{2}}{18 a^{3} H^{3}}\right)^{-1}$.

The solution of Eq. (27) is

$u(\phi)=-\int_{\phi_{*}}^{\phi} d \psi r(\psi) \exp \left(-\int_{\psi}^{\phi} Q(X) d X\right)$,

where $\phi_{*}$ is chosen to satisfy proper boundary conditions.

Then (an analog of the formula derived by Starobinsky and Vennin [23])

$f(\phi)=-\int_{\phi_{1}}^{\phi} d \phi^{\prime} \int_{\phi_{*}}^{\phi^{\prime}} d \psi r(\psi) \exp \left(-\int_{\psi}^{\phi^{\prime}} Q(X) d X\right)$.

This solution satisfies $f\left(\phi_{1}\right)=0$ and $\phi_{*}$ is chosen so that $f\left(\phi_{2}\right)=0$. Then, according to Eq. (26)(setting $\phi=\phi_{2}$ to get $f\left(\phi_{2}\right)=0$ ) we have (an approximate formula for a mean value of e-folds appeared already in [11])

$\langle v\rangle=-\int_{\phi_{1}}^{\phi_{i n}} d \phi^{\prime} \int_{\phi_{*}}^{\phi^{\prime}} d \psi r(\psi) \exp \left(-\int_{\psi}^{\phi^{\prime}} Q(X) d X\right)$.

$v$ is the umber of e-folds between $\phi_{\text {in }}$ and $\phi_{\text {end }}=\phi_{1}$. We have to determine $\phi_{*}$ from the condition $f\left(\phi_{2}\right)=0$.

Another method is considered in [26]. There, the solution of Eq. (27) is written in the form

$$
\begin{aligned}
u(\phi)= & \exp \left(-\int_{\phi_{*}}^{\phi} Q(X) d X\right) u\left(\phi_{*}\right) \\
& -\int_{\phi_{*}}^{\phi} d \psi r(\psi) \exp \left(-\int_{\psi}^{\phi} Q(X) d X\right)
\end{aligned}
$$

Then, the boundary conditions are expressed by $u\left(\phi_{*}\right)$ and $\phi_{*}$. Integrating Eq. (33)

$$
\begin{aligned}
f(\phi)= & \int_{\phi_{1}}^{\phi} d \phi^{\prime} \exp \left(-\int_{\phi_{*}}^{\phi^{\prime}} Q\right) u\left(\phi_{*}\right) \\
& -\int_{\phi_{1}}^{\phi} d \phi^{\prime} \int_{\phi_{*}}^{\phi^{\prime}} d \psi r(\psi) \exp \left(-\int_{\psi}^{\phi^{\prime}} Q\right) .
\end{aligned}
$$

We demand

$$
\begin{aligned}
0= & f\left(\phi_{2}\right)=\int_{\phi_{1}}^{\phi_{2}} d \phi^{\prime} \exp \left(-\int_{\phi_{*}}^{\phi^{\prime}} Q\right) u\left(\phi_{*}\right) \\
& -\int_{\phi_{1}}^{\phi_{2}} d \phi^{\prime} \int_{\phi_{*}}^{\phi^{\prime}} d \psi r(\psi) \exp \left(-\int_{\psi}^{\phi^{\prime}} Q\right)
\end{aligned}
$$

Solving for $u\left(\phi_{*}\right)$ gives the formula for $v$

$$
\begin{aligned}
\langle v\rangle= & \left(\int_{\phi_{1}}^{\phi_{i n}} d \phi^{\prime} \exp \left(-\int_{\phi_{*}}^{\phi^{\prime}} Q\right)\right. \\
& \times \int_{\phi_{1}}^{\phi_{2}} d \phi^{\prime} \int_{\phi_{*}}^{\phi^{\prime}} d \psi r(\psi) \exp \left(-\int_{\psi}^{\phi^{\prime}} Q\right) \\
& -\int_{\phi_{1}}^{\phi_{2}} d \phi^{\prime} \exp \left(-\int_{\phi_{*}}^{\phi^{\prime}} Q\right) \int_{\phi_{1}}^{\phi_{i n}} d \phi^{\prime} \int_{\phi_{*}}^{\phi^{\prime}} d \psi r(\psi) \\
& \left.\times \exp \left(-\int_{\psi}^{\phi^{\prime}} Q\right)\right) \\
& \times\left(\int_{\phi_{1}}^{\phi_{2}} d \phi^{\prime} \exp \left(-\int_{\phi_{*}}^{\phi^{\prime}} Q\right)\right)^{-1} .
\end{aligned}
$$

$\phi_{*}, \phi_{1}, \phi_{2}$ are arbitrary but a useful choice is $\phi_{1}=\phi_{\text {end }}$, $\phi_{2}=\infty$ and $\phi_{*}=\phi_{i n}$. So, we calculate the first hitting of a boundary of an interval $\left[\phi_{\text {end }}, \infty\right]$ by the process starting from $\phi_{\text {in }}$ (we know that $\phi=\infty$ cannot be achieved, so there remains $\left.\phi_{\text {end }}\right)$. There may be some problems with integrability in Eq. (36) with some potentials in an infinite interval $[24,25]$ (if there is no thermal noise). Then, $\int Q \simeq-V^{-1}$ and the integrability may fail if either $V=0$ or $V$ does not grow fast enough.

From the formula (23) we have

$$
\begin{aligned}
v= & f_{I}\left(\phi_{i n}\right)+\int_{0}^{\phi} \partial_{\phi} f_{I}\left(\frac{\gamma}{3(a H)^{\frac{3}{2}}} d B(v)\right. \\
& \left.+\frac{1}{2 \pi} H d W(v)\right) .
\end{aligned}
$$

Taking the square and then the expectation value of Eq. (37) we obtain

$$
\begin{aligned}
\left\langle v^{2}\right\rangle & \equiv\langle v\rangle^{2}+\left\langle(\delta v)^{2}\right\rangle \\
& =\langle v\rangle^{2}+\left\langle\int_{0}^{v} d v^{\prime}\left(\partial_{\phi} f_{I}\right)^{2}\left(\frac{\gamma^{2}}{9(a H)^{3}}+\frac{H^{2}}{4 \pi^{2}}\right)\right\rangle
\end{aligned}
$$

(an analogous formula holds true for $f_{S}$ ). Assume that we find a function $F_{S}$ such that

$$
\begin{aligned}
-\partial_{\phi} & F_{S} \frac{1}{3 H^{2}} V^{\prime}+\frac{1}{18} \frac{\gamma}{(a H)^{\frac{3}{2}}} \partial_{\phi} \frac{\gamma}{(a H)^{\frac{3}{2}}} \partial_{\phi} F_{S} \\
+ & \frac{1}{8 \pi^{2}} \partial_{\phi} H \partial_{\phi} H F_{S} \\
= & -\left(\partial_{\phi} f_{S}\right)^{2}\left(\frac{\gamma^{2}}{9(a H)^{3}}+\frac{H^{2}}{4 \pi^{2}}\right)
\end{aligned}
$$

in the Stratonovitch case and $F_{I}$

$$
\begin{gathered}
-\partial_{\phi} F_{I} \frac{1}{3 H^{2}} V^{\prime}+\frac{1}{18}\left(\frac{\gamma}{(a H)^{\frac{3}{2}}}\right)^{2} \partial_{\phi} \partial_{\phi} F_{I}+\frac{1}{8 \pi^{2}} H^{2} \partial_{\phi} \partial_{\phi} F_{I} \\
=-\left(\partial_{\phi} f_{I}\right)^{2}\left(\frac{\gamma^{2}}{9(a H)^{3}}+\frac{H^{2}}{4 \pi^{2}}\right)
\end{gathered}
$$


in the Ito case. Then, calculating $d F$ in the same way as we did for $d f$ in Eqs. (22) and (23) using Eqs. (38)-(40) and taking the expectation value we find

$\left\langle v^{2}\right\rangle-\langle v\rangle^{2}=F\left(\phi_{i n}\right)$.

Let us denote $U=\partial_{\phi} F_{I}$. Then, in the Ito version we have the equation

$\partial_{\phi} U+Q U=-R$

with $Q$ defined in Eq. (28) and

$R=2\left(\partial_{\phi} f_{I}\right)^{2}$.

The solution follows the one of Eq. (27) as expressed either in Eq. (30) or in Eq. (33). In the next section we discuss perturbative solutions of Eqs. (27) and (42).

\section{4 e-folds, their fluctuations and the power spectrum}

The general integral formulae in Sect. 3 do not allow an explicit calculation of the functions $F$ and $f$ needed for a computation of the e-folds and their fluctuations. We need a perturbative approach. We write Eq. (27) as an iterative perturbation expansion in $\frac{1}{Q}$ starting with $u^{(1)}=-\frac{r}{Q}$

$u^{(n+1)}=-\frac{1}{Q} \partial_{\phi} u^{(n)}$.

Then, the zeroth order approximation in Eq. (27) corresponds to setting $\frac{1}{Q} \partial_{\phi} u=0$. Hence,

$u^{(1)}=f^{\prime}=-r Q^{-1}=8 \pi G V\left(V^{\prime}\right)^{-1}$.

In this approximation we have derived the "classical" formula (9) for e-folds. We have

$\partial_{\phi} u^{(1)}=8 \pi G\left(1-\frac{\eta}{2 \epsilon}\right)$

with

$\epsilon=\frac{1}{16 \pi G}\left(\frac{V^{\prime}}{V}\right)^{2}$

$\eta=\frac{1}{8 \pi G} \frac{V^{\prime \prime}}{V}$.

Hence, $\partial_{\phi} u^{(1)} \neq 0$ in general. We get $\partial_{\phi} u^{(1)}=0$ for the exponential potential. For a power-law potential $\phi^{n}$ we have $\partial_{\phi} u^{(1)}=\frac{8 \pi G}{n} \cdot u^{(1)}$ is independent of $\gamma$ but $u^{(2)}$ (44) depends on $\gamma$ as $Q$ does. If the thermal noise is absent then the expansion (44) is an expansion in $G$ (i.e., in the inverse of the Planck mass). Next, we need an approximation for the solution of Eq. (42). Applying again the expansion (44) in $\frac{1}{Q}$ (in the lowest order $\frac{1}{Q} \partial_{\phi} U \simeq 0$ ) we obtain

$$
\begin{aligned}
U^{(1)} & =\partial_{\phi} F^{I}=-R Q^{-1} \\
& =6(8 \pi G)^{2} V^{2}\left(V^{\prime}\right)^{-3} H^{2}\left(\frac{H^{2}}{8 \pi^{2}}+\frac{\gamma^{2}}{18 a^{3} H^{3}}\right) .
\end{aligned}
$$

In the next order

$U^{(2)}=3 H^{2}\left(\frac{H^{2}}{8 \pi^{2}}+\frac{\gamma^{2}}{18 a^{3} H^{3}}\right)\left(V^{\prime}\right)^{-1} \partial_{\phi} U^{(1)}$.

The power spectrum $\mathcal{P} \simeq U u^{-1}$ at $\gamma=0$ derived from Eqs. (45) and (49) coincides with the standard formula [37,39-42]. It can be obtained from the general formulae of Sect. 3 which involve $\int Q$. These formulae for e-foldings in the quantum case (cold inflation) have been discussed by Starobinsky and Vennin [23]. If $\gamma=0$ then

$\int_{\psi}^{\phi} Q=-\frac{3}{8 G^{2}} \frac{1}{V(\phi)}+\frac{3}{8 G^{2}} \frac{1}{V(\psi)}$.

In order to calculate the integrals (30)-(36) they perform the Taylor expansion of $\frac{1}{V(\psi)}$ around $\phi$

$\frac{1}{V(\psi)}=\frac{1}{V(\phi)}+\partial_{\phi} \frac{1}{V(\phi)}(\psi-\phi)+\cdots$

Changing variables

$\psi-\phi=G^{2} X$

and expanding the exponential in $G^{2}$ we derive the perturbation expansion (44).

Next, let us consider the thermal noise using the method of Starobinsky and Vennin $[4,23,38]$ (which is equivalent to the expansion (44) in $\gamma^{2} G^{-\frac{1}{2}}$ and in $G$ ). Then, in Eq. (28) (without the quantum noise)

$Q=-\frac{6}{\gamma^{2}} \sqrt{\frac{8 \pi G}{3}} V^{\prime} \sqrt{V} a^{3}$

and in Eq. (29)

$r=\gamma^{-2} 48 \pi G\left(\frac{3}{8 \pi G}\right)^{\frac{1}{2}} V^{\frac{3}{2}} a^{3}$

We have by an integration by parts

$$
\begin{aligned}
\int_{\psi}^{\phi} Q= & -\frac{4}{\gamma^{2}} \sqrt{\frac{8 \pi G}{3}}\left(a^{3}(\phi) V^{\frac{3}{2}}(\phi)\right. \\
& \left.-a^{3}(\psi) V^{\frac{3}{2}}(\psi)\right)-\frac{32 \pi G}{\gamma^{2}} \sqrt{\frac{8 \pi G}{3}} \int_{\psi}^{\phi} \frac{V^{\frac{5}{2}} a^{3}}{V^{\prime}} .
\end{aligned}
$$

Let

$\Omega(\psi)=\frac{4}{\gamma^{2}} \sqrt{\frac{8 \pi G}{3}} a^{3}(\psi) V^{\frac{3}{2}}(\psi)$.

We repeat the approximation (51) used in $[23,38]$ when applied to Eq. (31). We expand in Eq. (31) the exponential function 
$\Omega(\psi)=\Omega(\phi)+\partial_{\phi} \Omega(\phi)(\psi-\phi)+\cdots$

Then, in the integral (31) we have (neglecting $\partial_{\phi} a \simeq G$ and the second term in Eq. (54) being of higher order in $G$ )

$$
\begin{aligned}
\langle v\rangle & =\int d \phi r(\phi) \int^{\phi} d \psi \exp \left(\partial_{\phi} \Omega(\phi) \psi\right) \\
& \simeq 8 \pi G \int d \phi \frac{V}{V^{\prime}}
\end{aligned}
$$

By means of integral formulae of Sect. 3 as well as with the perturbation expansion (44) we obtain in the first order the same formula for e-folds as we could get in the calculation without noise (showing that the stochastic method of reaching the boundary has the correct no noise limit; the stochastic formula will still be discussed in Appendix A). The calculation of $\left\langle\delta v^{2}\right\rangle$ with the thermal noise on the basis of Eq. (41) involves calculation of the integral

$F \simeq 2 \int^{\phi} d \psi\left(f^{\prime}(\psi)\right)^{2} \exp \left(-\int_{\psi}^{\phi} Q\right)$

with $Q$ of Eq. (52). The Taylor expansion (56) in the integral (58) gives

$U=F^{\prime}=-\gamma^{2}(8 \pi G)^{2}(24 \pi G)^{-\frac{1}{2}} V^{\frac{3}{2}}\left(V^{\prime}\right)^{-3} a^{-3}$.

The power spectrum can be defined by fluctuations of the e-folds

$\mathcal{P}=\frac{d\left\langle(\delta v)^{2}\right\rangle}{d\langle v\rangle}$

where $\left\langle(\delta v)^{2}\right\rangle$ is defined in Eq. (38). We have

$\frac{d}{d\langle\nu\rangle}=-\left(f^{\prime}\right)^{-1} \frac{d}{d \phi}$.

Hence

$\mathcal{P}=F^{\prime}\left(f^{\prime}\right)^{-1} \equiv \frac{U}{u}$

(evaluated at the horizon crossing $k=a H$ [43]) where $f$ is defined in Eqs. (24) and (25) and $F$ in Eqs. (39) and (40) (calculated from Eq. (42)). It has been shown [23,38] that the formula (62) in the expansion (51) (no thermal noise) coincides with the standard one for the cold inflation [37,33,39-43] because we obtain from Eqs. (41), (42), (45) and (51)

$\mathcal{P}_{q}=\frac{2 G^{2}}{3} 8 \pi G V^{3}\left(V^{\prime}\right)^{-2}$.

It is difficult to calculate $\mathcal{P}$ analytically for general quantum and thermal noise using the formulae of Sect. 3. We calculate the power spectrum with no quantum noise (solely thermal noise) applying for $F^{\prime}$ the same approximation which we used in Eq. (62) (for $\langle v\rangle$, i.e. for $f^{\prime}$ ). Then, from Eq. (63)

$\mathcal{P}_{t h}=8 \pi G \gamma^{2}(24 \pi G)^{-\frac{1}{2}} a^{-3} V^{\frac{1}{2}}\left(V^{\prime}\right)^{-2}$.
From the $\frac{1}{Q}$ expansion using Eqs. (45), (49) and (62) we obtain

$\mathcal{P}=\mathcal{P}_{t h}+\mathcal{P}_{q}$

(this simple additivity holds true only in the lowest order of the $\frac{1}{Q}$ expansion as can be seen from Eq. (50)). The spectral index $n_{S}$ can be calculated as a derivative (61) over $\langle v\rangle$ of $\ln \mathcal{P}$. Then

$n_{S}-1=-\left(f^{\prime}\right)^{-1} \frac{d}{d \phi} \ln \mathcal{P}=-F^{\prime \prime}\left(f^{\prime} F^{\prime}\right)^{-1}+f^{\prime \prime}\left(f^{\prime}\right)^{-2}$

We obtain from Eq. (64) (the spectral index for warm inflation is calculated in $[44,45]$ but under different assumptions)

$n_{S}^{t h}-1=-3-\epsilon+2 \eta$.

For the quantum stochastic inflation the formula (63) gives

$n_{S}^{q}-1=-6 \epsilon+2 \eta$

(in agreement with [39-43]). On the basis of the $\frac{1}{Q}$ expansion using Eqs. (66) and (65) we obtain for the spectral index of the scalar field in thermal and quantum noises

$n_{S}-1=\left(\mathcal{P}_{q}+\mathcal{P}_{t h}\right)^{-1}\left(\mathcal{P}_{q}\left(n_{S}^{q}-1\right)+\left(n_{S}^{t h}-1\right) \mathcal{P}_{t h}\right)$.

For a small $\gamma$ from Eq. (69) and Eqs. (63) and (64)

$n_{S}-1=\left(\left(n_{S}^{q}-1\right)+\frac{32 \pi^{2} \gamma^{2}}{9} H^{-5} a^{-3}\left(n_{S}^{t h}-n_{S}^{q}\right)\right)$.

\section{Summary}

The method of a description of inflation in terms of the fluctuations of e-folds (called $\delta N$ method) has been suggested long time ago $[4,41,46]$, developed recently and applied to detailed estimates of inflation parameters [23-25,47-51]. In this paper we have extended this formalism to include a thermal noise. The thermal noise modifies the results on power spectrum. In principle, the method allows to calculate the inflationary parameters non-perturbatively for a larger class of potentials. In the lowest order of a perturbative expansion we have obtained a formula for the power spectrum which is just a sum of the density of thermal (proportional to $\gamma^{2}$ ) and quantum fluctuations. The spectral index is an average of spectral indices of thermal and quantum fluctuations with the corresponding power spectra. The correction to the power spectrum is proportional to $\gamma^{2}$ (which is small for low temperature in the warm inflation models). The spectral index depends on the inflaton potential. It is measurable in observations [1]. Its value can give some information on the inflaton potential as well as on the friction in inflaton wave equation. 
Acknowledgements The author thanks the anonymous referees whose comments contributed to a substantial improvement of the initial version of this paper.

Open Access This article is distributed under the terms of the Creative Commons Attribution 4.0 International License (http://creativecomm ons.org/licenses/by/4.0/), which permits unrestricted use, distribution, and reproduction in any medium, provided you give appropriate credit to the original author(s) and the source, provide a link to the Creative Commons license, and indicate if changes were made. Funded by SCOAP ${ }^{3}$.

\section{Appendix A}

It is instructive to compare approximations applied in Sects. 3-5 with exact solutions (some solutions of stochastic equations with quantum noise are discussed in [16-19]).

For the exponential potential (see [34,52]) $V=g \exp (\lambda \phi)$ in the e-folding time the stochastic equation (6) reads (with $a(\phi)$ derived in Eq. (16))

$$
\begin{aligned}
d \phi= & -\frac{\lambda}{8 \pi G} d \nu+\frac{1}{2 \pi} \sqrt{\frac{8 \pi G g}{3}} \exp \left(\frac{1}{2} \lambda \phi\right) \circ d W(\nu) \\
& +\frac{\gamma}{3}\left(\frac{3}{8 \pi G g}\right)^{\frac{3}{4}} \exp (\alpha \phi) \circ d B(\nu),
\end{aligned}
$$

where

$\alpha=\frac{12 \pi G}{\lambda}-\frac{3}{4} \lambda$

If we express $a$ by $v$ in Eq. (6) (leading to the Fokker-Planck equation (14)) then

$$
\begin{aligned}
d \phi= & -\frac{\lambda}{8 \pi G} d \nu+\frac{1}{2 \pi} \sqrt{\frac{8 \pi G g}{3}} \exp \left(\frac{1}{2} \lambda \phi\right) \circ d W(\nu) \\
& +\frac{\gamma}{3}\left(\frac{3}{8 \pi G g}\right)^{\frac{3}{4}} \exp \left(-\frac{3}{2} v\right) \exp \left(-\frac{3}{4} \lambda \phi\right) \circ d B(\nu) .
\end{aligned}
$$

In the Stratonovitch interpretation these equations can be solved exactly if either the quantum noise or the thermal noise are absent. No exact solution exists if the Stratonovitch differential in Eqs. (70) and (71) is replaced by the Ito differential. In such a case in order to approach the solution we would have to use the relation [30] (with a certain parameter $\sigma) \exp (\sigma \phi) d W=\exp (\sigma \phi) \circ d W-\frac{1}{2} \sigma d \phi d W \exp (\sigma \phi)$. The resulting Stratonovitch equation (which can be treated as an ordinary differential equation) would not be linear. If quantum noise is absent then we set

$$
X=\exp (-\alpha \phi) .
$$

Then (in the decomposition into classical and stochastic parts)

$$
\begin{aligned}
X \equiv & X_{c l}+X_{s t}=\exp \left(\frac{\alpha \lambda}{8 \pi G} v\right) X_{0} \\
& -\frac{\alpha \gamma}{3}\left(\frac{3}{8 \pi G g}\right)^{\frac{3}{4}} \int_{0}^{v} \exp \left(\frac{\alpha \lambda}{8 \pi G}(v-s)\right) d B(s) .
\end{aligned}
$$

For Eq. (71) we set

$$
\tilde{X}=\exp \left(\frac{3}{4} \lambda \phi\right)
$$

Then (no quantum noise)

$$
\begin{aligned}
\tilde{X}= & \exp \left(-\frac{3 \lambda^{2}}{32 \pi G} v\right) \tilde{X}_{0}+\frac{\lambda \gamma}{4} \\
& \times\left(\frac{3}{8 \pi G g}\right)^{\frac{3}{4}} \int_{0}^{v} \exp \left(-\frac{3 \lambda^{2}}{32 \pi G}(\nu-s)-\frac{3}{2} s\right) d B(s) .
\end{aligned}
$$

If $\gamma=0$ in Eq. (70) then we set

$$
Y=\exp \left(-\frac{1}{2} \lambda \phi\right)
$$

We obtain the solution

$$
\begin{aligned}
Y \equiv & Y_{c l}+Y_{s t}=\exp \left(\frac{\lambda^{2}}{16 \pi G} v\right) Y_{0} \\
& -\frac{\lambda}{4 \pi} \sqrt{\frac{8 \pi G g}{3}} \int_{0}^{v} \exp \left(\frac{\lambda^{2}}{16 \pi G}(v-s)\right) d W(s) .
\end{aligned}
$$

We can calculate the power spectrum from the formula for the energy density fluctuations $\delta \rho$

$$
\left\langle\left(\frac{\delta \rho}{\rho}\right)^{2}\right\rangle=\left\langle\left(H\left(\frac{d \phi}{d t}\right)^{-1} \delta \phi\right)^{2}\right\rangle,
$$

where

$\delta \phi=\phi-\phi_{c l}$.

For the exponential interaction in the slow-roll approximation

$$
\left(\frac{H}{\frac{d \phi}{d t}}\right)^{2}=(8 \pi G)^{2} \lambda^{-2} .
$$

Hence, fluctuations of $\frac{\delta \rho}{\rho}$ are proportional to fluctuations of $\phi$. Fluctuations of $\phi$ in Eqs. (73), (75) and (77) can be calculated in a power series expansion in the noise (see similar calculations in [53]). So, in the case of the thermal noise 
$\phi-\phi_{c l}=\beta \int_{0}^{\nu} d s \exp \left(-\frac{\lambda \alpha s}{8 \pi G}\right) d B(s)$

where

$\beta=\frac{\gamma}{3}\left(\frac{3}{8 \pi G g}\right)^{\frac{3}{4}}$

Hence,

$\left\langle(\delta \phi)^{2}\right\rangle=\left(\frac{4 \beta \pi G}{\alpha}\right)^{2}\left(1-\exp \left(-\frac{\lambda \alpha \nu}{4 \pi G}\right)\right)$.

In the case of the quantum noise

$\left\langle(\delta \phi)^{2}\right\rangle=\frac{4}{3} \lambda^{-2}(8 \pi G)^{2}\left(1-\exp \left(-\frac{\lambda^{2} \nu}{8 \pi G}\right)\right)$

(in agreement with Eqs. (38), (39) of [22]). $v$ should be taken at the time $v \simeq-\ln k$ expressed by the wave number $k$ at the Hubble horizon crossing.

From Eq. (9)

$v=v_{i n}-\frac{8 \pi G}{\lambda} \phi$.

Hence, at the lowest order fluctuations of $\phi$ are proportional to fluctuations of $v$. Now, we can calculate the spectral index as $\partial_{v} \ln \left\langle(\delta \phi)^{2}\right\rangle$ with the result

$n_{S}^{t h}-1=-3+\frac{3 \lambda^{2}}{16 \pi G}$

for thermal noise and

$n_{S}^{q}-1=-\frac{\lambda^{2}}{8 \pi G}$

for the Starobinsky (quantum) noise in agreement with Eqs. (67) and (68). In all cases (73)-(77) we obtain stochastic corrections to the classical formula (9). As an example from Eq. (77)

$$
\begin{gathered}
\left\langle\exp \left(-\frac{1}{2} \lambda \phi\right)\right\rangle \simeq\left\langle\exp \left(-\frac{1}{2} \lambda \phi_{c l}\right)\right\rangle \\
=\exp \left(-\frac{1}{2} \lambda \phi_{0}\right)\left\langle\exp \left(\frac{\lambda^{2}}{16 \pi G} v\right)\right\rangle .
\end{gathered}
$$

Hence, in the approximation $\langle\exp (f)\rangle \simeq \exp (\langle f\rangle)$ we obtain the classic formula $\langle v\rangle=-8 \pi G\langle\phi\rangle$. From Eq. (77) we could obtain further relations between correlation functions of $\phi$ and $v$.

\section{Appendix B}

Let us repeat the derivation of the fluctuation equations (without the thermal noise) of Refs. [21-23]with some care concerning the rules of the stochastic calculus $[30,54]$. We discuss here also the difference between the Ito and Stratonovith versions. The conventional rules of the differential calculus (in particular, the Leibniz rule) are satisfied in the
Stratonovitch form of stochastic equations. On the other hand correlation functions are easier to calculate with the Ito integrals (in particular, an expectation value of the Ito integral is zero). One can relate both integrals according to the rule

$f \circ d W=f d W+\frac{1}{2} d f d W$,

where after calculation of $d f$ as a function of $W$ we use the rule $d W d W=d \nu$.

Let

$\partial_{\nu} \phi_{c l}=-\frac{1}{3} H\left(\phi_{c l}\right)^{-2} V^{\prime}\left(\phi_{c l}\right)$,

where $H$ is related to $V$ by Eq. (8). Let $\delta=\phi-\phi_{c}$. First, consider the Stratonovitch version of Eq. (6). We write $\phi=$ $\phi_{c}+\delta$, use Eq. (88) and expand Eq. (6) till the second order in $\delta$. Then, integrating, taking the expectation value and using $\left\langle\int f d W\right\rangle=0$ we obtain

$$
\begin{aligned}
\langle\delta\rangle^{\prime}= & \left(\left(\ln \left(\frac{H^{\prime}}{H}\right)\right)^{\prime}-\frac{G}{2 \pi}\left(H^{\prime} H\right)^{\prime} \frac{H}{H^{\prime}}\right)\langle\delta\rangle \\
& +\frac{H}{H^{\prime}}\left(\frac{1}{2}\left(\frac{H^{\prime}}{H}\right)^{\prime \prime}-\frac{G}{4 \pi}\left(H H^{\prime}\right)^{\prime \prime}\right)\left\langle\delta^{2}\right\rangle-\frac{G}{2 \pi} H^{2} .
\end{aligned}
$$

The equation for fluctuations reads

$$
\begin{aligned}
\left\langle\delta^{2}\right\rangle^{\prime}= & \left(2\left(\ln \left(\frac{H^{\prime}}{H}\right)\right)^{\prime}-\frac{G}{\pi}\left(H^{2}\right)^{\prime \prime} \frac{H}{H^{\prime}}\right)\left\langle\delta^{2}\right\rangle \\
& -\frac{3 G}{\pi} H^{2}\langle\delta\rangle-\frac{G}{\pi} \frac{H^{3}}{H^{\prime}} .
\end{aligned}
$$

In these equations we have replaced the variable $v$ by $\phi_{c l}$ on the basis of Eq. (88). The "prime" denotes a differentiation with respect to $\phi_{c l}$.

In the Ito interpretation of Eq. (6)

$$
\begin{aligned}
\langle\delta\rangle^{\prime}= & \left(\ln \left(\frac{H^{\prime}}{H}\right)\right)^{\prime}\langle\delta\rangle+\frac{1}{2} \frac{H}{H^{\prime}}\left(\frac{H^{\prime}}{H}\right)^{\prime \prime}\left\langle\delta^{2}\right\rangle, \\
\left\langle\delta^{2}\right\rangle^{\prime}= & \left(2\left(\ln \left(\frac{H^{\prime}}{H}\right)\right)^{\prime}-\frac{G}{2 \pi}\left(H^{2}\right)^{\prime \prime} \frac{H}{H^{\prime}}\right)\left\langle\delta^{2}\right\rangle \\
& -\frac{2 G}{\pi} H^{2}\langle\delta\rangle-\frac{G}{\pi} \frac{H^{3}}{H^{\prime}} .
\end{aligned}
$$

Equation (92) is different from Eq. (38) of [21] and Eq. (A.22) of [23] as the terms $G H^{2}\langle\delta\rangle$ and $G\left(H^{2}\right)^{\prime \prime}$ are absent there. We can solve both Stratonovitch (90)-(89) and Ito (91)-(92) equations in a perturbation expansion in $m_{p l}^{-2}=8 \pi G$. We expand the solution of Eqs. (91) and (92) around the one of $[21,23]$ (with $\langle\delta\rangle=0$ and no extra terms) then we obtain that in such an expansion $\langle\delta\rangle \simeq G^{2}$. Hence, the term $G H^{2}\langle\delta\rangle$ in Eq. (92) will be of order $G^{4}$. As $\left\langle\delta^{2}\right\rangle \simeq G^{2}$ another extra term in Eq. (92) $G\left(H^{2}\right)^{\prime \prime}\left\langle\delta^{2}\right\rangle \simeq G^{4}$. Hence, in comparison with Refs. [21,23] the extra terms are of higher order in $G$ (at this 
order, our starting point, the equations for stochastic inflation would also need a modification). The same argument applies to the Stratonovitch Eq. (90). It is different from equations of Refs. [21,23] by terms of order $G^{4}$. For this reason till the order $G^{4}$ we have the same conclusions concerning the solution of Eqs. (90) or (92) (these equations determine the fluctuations and power spectra).

We can repeat the calculations of fluctuations for the thermal noise rederiving the formula (64). Then, at the lowest order in $\gamma^{2}$ we get the additivity of fluctuations (65) and as a consequence the formula (69) for the spectral index.

Let us consider the particular case of the Stratonovitch stochastic equation (6) for $V=\frac{1}{2} m^{2} \phi^{2}$ with no thermal noise

$d \phi=-\frac{d v}{4 \pi G \phi}+q \phi \circ d W(v)$,

where $q=\sqrt{\frac{G m^{2}}{3 \pi}}$. The Ito version of Eq. (6) is

$$
\begin{aligned}
d \phi= & -\frac{d v}{4 \pi G \phi}+q \phi d W(v)=-\frac{d v}{4 \pi G \phi}-\frac{1}{2} q^{2} \phi d v \\
& +q \phi \circ d W(v)
\end{aligned}
$$

Equation (93) has the solution

$$
\begin{aligned}
\phi_{v}^{2}= & \phi_{0}^{2} \exp (2 q W(v)) \\
& -\frac{1}{2 \pi G} \int_{0}^{v} d s \exp (2 q W(v)-2 q W(s))
\end{aligned}
$$

where $\phi_{0}$ is the initial value. The solution of Eq. (94) reads

$$
\begin{aligned}
\phi_{v}^{2}= & \phi_{0}^{2} \exp \left(-q^{2} v+2 q W(v)\right) \\
& -\frac{1}{2 \pi G} \int_{0}^{v} d s \exp \left(-q^{2}(v-s)+2 q W(v)-2 q W(s)\right) .
\end{aligned}
$$

We can express correlation functions of $\phi^{2}$ in terms of correlations of $\nu$. In particular, for the Stratonovitch version

$\left\langle\phi_{\nu}^{2}\right\rangle=\left(\phi_{0}^{2}-q^{-2} \frac{1}{4 \pi G}\right)\left\langle\exp \left(2 q^{2} v\right)\right\rangle+q^{-2} \frac{1}{4 \pi G}$

and for the Ito version

$\left\langle\phi_{\nu}^{2}\right\rangle=\left(\phi_{0}^{2}-q^{-2} \frac{1}{2 \pi G}\right)\left\langle\exp \left(q^{2} v\right)\right\rangle+q^{-2} \frac{1}{2 \pi G}$.

It can be seen that from the requirement of positivity of $\phi_{v}^{2}$ we get some bounds on the initial values $\phi_{0}$ and expectation values of $v$. In particular, the relation (15) $\left\langle\phi_{\nu}^{2}\right\rangle=$ const $-\frac{1}{2 \pi G}\langle\nu\rangle$ holds true only in the lowest order in $q$. The fluctuation equations for the model $\frac{1}{2} m^{2} \phi^{2}$ discussed in [23, Eq. (A.33)] follow from Eqs. (90) or (92). The exact solution (95) and (96) does not tell us more than Eqs. (90)-(92) (with $H \simeq \phi$ ) concerning quadratic fluctuations. However, using the solutions (95) and (96) we could get explicitly the higher order fluctuations of $\phi^{2}$.

\section{References}

1. P.A.R. Ade et al., arXiv: 1502.01589

2. A.A. Starobinsky, JETP Lett. 30, 682 (1979)

3. V.F. Mukhanov, G.V. Chibisov, JETP Lett. 33, 532 (1981)

4. A.A. Starobinsky, Phys. Lett. B 117, 175 (1982)

5. S.W. Hawking, Phys. Lett. B 115, 295 (1982)

6. A.H. Guth, S.Y. Pi, Phys. Rev. Lett. 49, 1110 (1982)

7. J.M. Bardeen, P.J. Steinhardt, M.S. Turner, Phys. Rev. D 28, 678 (1985)

8. A. Berera, Phys. Rev. D 54, 2519 (1996)

9. Z. Haba, Adv. Energy Phys. 2018, 7204952 (2018)

10. A. Berera, I.G. Moss, R.O. Ramos, Rep. Prog. Phys. 72, 026901 (2009)

11. A.A. Starobinsky, in Current Topics in Field Theory, Quantum Gravity and Strings, vol. 246, Lecture Notes in Phys., ed. by H.J. Vega, N. Sanchez (Springer, New York, 1986), pp. 107-126

12. A. Vilenkin, Phys. Rev. D 27, 2848 (1983)

13. A.D. Linde, Phys. Rev. D 58, 083514 (1998)

14. A.D. Linde, Phys. Rev. D 49, 748 (1994)

15. S. Mattarrese, A. Ortolan, F. Lucchin, Phys. Rev. D 40, 290 (1989)

16. Y. Nambu, Prog. Theor. Phys. 81, 1037 (1989)

17. S. Gratton, N. Turok, Phys. Rev. D 72, 043507 (2005)

18. Y. Yi, E.T. Vishniac, Phys. Rev. 47, 5280 (1993)

19. J. Martin, M. Musso, Phys. Rev. D 73, 043516 (2006)

20. A. Vilenkin, Phys. Rev. D 59, 123506 (1999)

21. F. Finelli, G. Marozzi, A.A. Starobinsky, G.P. Vacca, G. Venturi, Phys. Rev. D 79, 044007 (2009)

22. F. Finelli, G. Marozzi, A.A. Starobinsky, G.P. Vacca, G. Venturi, Phys. Rev. D 82, 064020 (2010)

23. V. Vennin, A.A. Starobinsky, Eur. Phys. J. C 75, 413 (2015)

24. V. Vennin, H. Assadullahi, H. Firouzjahi, M. Noorbala, D. Wands, Phys. Rev. Lett. 118, 031301 (2017)

25. H. Assadullahi, H. Firouzjahi, M. Noorbala, D. Wands, JCAP 06, $043(2016)$

26. I.I. Gikhman, A.B. Skorohod, Stochastic Differential Equations (Springer, Berlin, 1972)

27. Z. Haba, arXiv:1802.00841 (subm. to Int. J. Mod. Phys. D)

28. Z. Haba, A. Stachowski, M. Szydlowski, JCAP 07, 024 (2016)

29. T. Josset, A. Perez, D. Sudarsky, Phys. Rev. Lett. 118, 021102 (2017)

30. N. Ikeda, S. Watanabe, Stochastic Differential Equations and Diffusion Processes (North Holland, Amsterdam, 1981)

31. P. Steinhardt, M.S. Turner, Phys. Rev. D 29, 2162 (1984)

32. A. Berera, Li-Zhi Fang, Phys. Rev. Lett. 74, 1912 (1995)

33. Jai-chan Hwang, Phys. Rev. D 48, 3544 (1993)

34. G. Marozzi, Phys. Rev. D 76, 043504 (2007)

35. H. Risken, The Fokker-Planck Equation (Springer, Berlin, 1989)

36. A. Linde, Phys. Lett. B 129, 177 (1983)

37. K. Freese, J.A. Frieman, A.V. Olinto, Phys. Rev. Lett. 65, 3233 (1990)

38. V. Vennin, PhD Thesis, https://tel.archives-ouvertes.fr/tel-0109 4199 (2014)

39. V.F. Mukhanov, Sov. Phys. JETP 68, 1297 (1988)

40. M. Sasaki, Prog. Theor. Phys. 76, 1036 (1986)

41. M. Sasaki, E.D. Stewart, Prog. Theor. Phys. 95, 71 (1996)

42. J.E. Lidsey, A.R. Liddle, E.W. Kolb, E.J. Copeland, T. Barreiro, M. Abney, Rev. Mod. Phys. 69, 374 (1997)

43. D.H. Lyth, A.R. Liddle, The Primordial Density Perturbation (Cambridge University Press, Cambridge, 2009)

44. M.H. Hall, I.G. Moss, A. Berera, Phys. Rev. D 69, 083525 (2004)

45. R.O. Ramos, L.A. da Silva, JCAP 03, 032 (2013)

46. A.A. Starobinsky, JETP Lett. 42, 152 (1985)

47. M. Liguori, S. Matarrese, M.A. Musso, A. Riotto, JCAP 08, 011 (2004) 
48. T. Fujita, M. Kawasaki, Y. Tada, T. Takesako, JCAP 12, 036 (2013)

49. T. Fujita, M. Kawasaki, Y. Tada, JCAP 10, 030 (2014)

50. R.J. Hardwick, V.Vennin, Ch.T. Byrnes, J. Torrado, D. Wands, arXiv: 1701.06473

51. Ch. Pattison, V. Vennin, H. Assadullahi, D. Wands, arXiv: 1707.00537
52. F. Lucchin, S. Matarrese, Phys. Rev. D 32, 1316 (1985)

53. A. Matacz, Phys. Rev. D 56, R1836 (1997)

54. B. Simon, Functional Integration and Quantum Mechaics (Academic Press, New York, 1979) 